\title{
A Wizard of Oz Study Exploring How Agreement/Disagreement Nonverbal Cues Enhance Social Interactions for Individuals Who Are Blind
}

\author{
Joshua Rader ${ }^{1}$, Troy McDaniel ${ }^{2}$, Artemio Ramirez Jr. ${ }^{3}$, \\ Shantanu Bala ${ }^{2}$, and Sethuraman Panchanathan ${ }^{2}$ \\ ${ }^{1}$ Hugh Downs School of Human Communication \\ Arizona State University, Tempe, AZ \\ ${ }^{2}$ Center for Cognitive Ubiquitous Computing \\ School of Computing, Informatics and Decision Systems Engineering \\ Arizona State University, Tempe, AZ \\ ${ }^{3}$ Department of Communication, University of South Florida, Tampa, FL \\ \{jbrader, troy.mcdaniel, shantanu.bala, panch\} @asu.edu, \\ aramirez@usf.edu
}

\begin{abstract}
Given their visual nature, nonverbal social cues, such as facial and head movements, are largely inaccessible to individuals who are blind, limiting the information gleaned during interactions. While social assistive aids have explored some nonverbal cues, such as detecting and communicating facial expressions, relatively few nonverbal cues have been explored. A thorough and systematic study has yet to investigate the importance and usefulness of many nonverbal social cues for individuals who are blind. This work takes this first step by beginning to explore the nonverbal cue of agreement/disagreement as indicated by head/body movements including head nod, head shake, leaning forward and leaning backward. To facilitate the investigation of the usefulness of nonverbal cues for individuals who are blind, we propose the use of a Wizard of $\mathrm{Oz}$ experiment to rapidly evaluate nonverbal communications using existing technologies rather than building new and complete systems. We first explore the usefulness of agreement/disagreement nonverbal cues using our existing Social Interaction Assistant platform in which most of the seemingly automated processes were manually performed by a wizard without the knowledge of participants. We conducted an experiment with 11 individuals who are blind or visually impaired involving one-on-one interactions with trained interviewers. Results show the potential of agreement/disagreement nonverbal cues within the social interactions of individuals who are blind.
\end{abstract}

Keywords: Social Interaction Assistant, social assistive aids, nonverbal communication, agreement, disagreement, haptic belt.

\section{Introduction}

Social interactions are an essential part of daily life that contribute to a person's general health and wellbeing. Social interactions are fundamental to friendships and other 
personal relationships; and have an indelible effect on who we are. However, there is limited access to the content conveyed during social interactions for those who are blind or visually impaired. Within a typical social interaction, $65 \%$ of the information exchanged is nonverbal, and $72 \%$ of nonverbal communication is visual [1]. Hence, individuals who are blind or visually impaired are severely limited in the amount of information they can glean from a social interaction. Furthermore, the inability to access facial expressions, body language, posture, and eye gaze can lead to potentially awkward and uncomfortable social situations for individuals who are blind. Overtime, situations such as these can lead to social avoidance and isolation.

While prior work [2-5] has developed, and to some extent, evaluated, social assistive aids for individuals who are blind, very few nonverbal cues have been investigated. Facial expressions are typically chosen for investigation, but nonverbal cues are extensive, rich and diverse, covering many other facets of nonverbal language. One possibility for the lack of a thorough and systematic study of the usefulness and importance of nonverbal cues for individuals who are blind might be the time and effort required to develop social assistive aids for various nonverbal cues. These technologies typically consist of sensing, processing and delivery components in which visual nonverbal cues are extracted using computer vision algorithms, and then relayed to the user through audio or haptic devices.

To avoid building new social assistive aids for each nonverbal cue to be communicated, we propose the use of a Wizard of $\mathrm{Oz}$ experiment to rapidly evaluate nonverbal cues for their usefulness and importance during social interactions involving individuals who are blind. A Wizard of $\mathrm{Oz}$ experiment [6] is an experimentation technique within the field of Human-Computer Interaction in which participants interact with a technology that is seemingly operating autonomously, but actually controlled completely or partially by the experimenter (wizard) without the knowledge of participants. Using this approach, we take the first step in exploring the usefulness of agreement/disagreement nonverbal cues for individuals who are blind. We use an existing Haptic Belt technology [5] in which head and body movements, specifically head nod, head shake, learning forward and learning backward, are manually recognized by the wizard, and subsequently sent to the haptic belt, delivering spatiotemporal vibrotactile patterns around the waist. We conducted an experiment with 11 individuals who are blind or visually impaired involving one-on-one interactions with trained interviewers. Results show the usefulness of a Wizard of Oz experimental approach, and the potential of agreement/disagreement nonverbal cues within the social interactions of individuals who are blind. The rest of the paper is structured as follows: Section 2 provides a review of social assistive aids. Section 3 introduces our proposed approach. Section 4 presents the results of a user study exploring the usability of the technology, and the relationship between nonverbal cues of agreement/disagreement and understanding. Section 5 concludes and provides possible directions for future research.

\section{Related Work}

Some prior work has explored social assistive aids that sense and deliver specific nonverbal cues to individuals who are blind including facial expressions [2, 3], interpersonal distance [4] and direction [5] of interaction partners. Rehman et al. [2] 
proposed a vibrotactile chair to convey emotions to individuals who are blind by mapping the manifold of facial expressions to the back of a chair. Their proposed vibrotactile display consisted of three axes along which convey the intensity of one of three emotions being visually expressed by an interaction partner: happy, sad or surprised. Krishna et al. [3] mapped the six basic emotions (happy, sad, surprised, angry, fear and disgust) to the back of the hand using a vibrotactile glove to convey emotions through spatiotemporal patterns inspired by both emoticons and affective haptics. McDaniel et al. [4] mapped interpersonal distances to tactile rhythms presented through a haptic belt using the analogy of a heartbeat: an increased "heart rate" conveys that interaction partners are becoming closer, indicative of intimacy. McDaniel et al. [5] have also explored the use of haptic belts for conveying both direction and distance of interaction partners to enhance the situational awareness of individuals who are blind during dyadic, or larger, social interactions.

While some progress has been made, many nonverbal cues have yet to be explored in the context of social assistive aids. In this work, we explore four nonverbal cues of agreement/disagreement [7] (head nod, head shake, leaning forward, leaning backward) and their usefulness to individuals who are blind. Bousmalis et al. [7] reference Poggi's [8] work to establish the three ways in which a person can express agreement/disagreement: Direct Speaker's Agreement/Disagreement, Indirect Speaker's Agreement/Disagreement, and Nonverbal Listener's Agreement/Disagreement. In this work, we focus exclusively on Nonverbal Listener's Agreement/Disagreement. Bousmalis et al. outline a full list of Nonverbal Listener's cues of agreement/disagreement noting that head nod is the most prevalent cue of agreement; and head shake is the most prevalent form of disagreement. It is also important to note that these two nonverbal cues (head nod, head shake) are generally agreed upon as indicating agreement and disagreement respectively [7, 8]. Nonverbal cues of learning forward/backward are useful for discerning agreement/disagreement, respectively, as indicative of interest (learning forward toward your interaction partner) and disinterest (learning away from your interaction partner). Bousmalis et al. establish a much needed set of accepted cues of agreement disagreement, but much work remains to develop and evaluate social assistive aids that convey these, among other, nonverbal cues in useful ways such that they complement social interactions.

To the best of the authors' knowledge, this is the first study to investigate how agreement/disagreement nonverbal cues may add to or enhance dyadic (one-on-one) interactions for individuals who are blind. The current study implements these nonverbal cues using existing technology, particularly a haptic belt [5] designed and built at the Center for Cognitive Ubiquitous Computing, through which the proposed visual head/body movements were mapped to spatiotemporal vibrotactile patterns around the waist.

\section{Proposed Approach}

To quickly evaluate the usefulness of nonverbal cues during social interactions for individuals who are blind, we propose the use of a Wizard of Oz experiment [6] to 
simulate (1) nonverbal cue extraction from an interaction partner through manual observation and recognition by an experimenter (wizard); and (2) delivery of the aforementioned nonverbal cues to the user (individual who is blind or visually impaired) by which the wizard manually sends the cues (using software) to an existing technology such as an audio or haptic device. In a Wizard of Oz experiment, participants use a technology that is seemingly operating autonomously, yet is partially or completely controlled by an experimenter (wizard). While deceptive, Wizard of $\mathrm{Oz}$ experiments help gather usability feedback quickly to save time and effort by avoiding the building of complete systems. Participants not knowing that parts of the system are simulated is critical to obtaining realistic feedback. Therefore, wizards should perform (1) and (2) outside of the experiment room to discreetly observe the interaction taking place via live video streams. Dyadic (one-on-one) interactions are recommended over larger interactions involving three or more people given the ease at which cues may be extracted and conveyed. Interactions should involve one individual who is sighted (interviewer), who is informed about the Wizard of Oz study and briefed on the discussion topics well in advanced; and one individual who is blind or visually impaired (interviewee) who will not know this is a Wizard of $\mathrm{Oz}$ study. The interviewer should be someone who is trained in human communication, wellarticulated and can carry a variety of discussion topics. The dyadic interaction may be semi-structured depending on the goals of the experiment. The mapping of nonverbal cues to the delivery technology will depend on the nonverbal cues being extracted, the technology being used, and the desired mapping between these modalities. In the next section, we describe our experiment exploring the usefulness of nonverbal cues related to agreement and disagreement conveyed through a haptic belt-an array of vibration motors placed around the waist.

\section{Experiment}

We conducted an Institutional Review Board (IRB)-approved experiment to examine the usefulness of nonverbal cues of agreement/disagreement in an interpersonal interaction for a person who is blind. In addition, the study explored the utility of the haptic belt as an effective means of relaying nonverbal communication. Utilizing previous research, we established four distinct vibrotactile cues to convey head and body movement that articulated nonverbal cues of agreement/disagreement: Head Shake: short vibrotactile pulses alternating at the left and ride sides of the waist (Left: $100 \mathrm{~ms}$ pulse, 150 ms gap, Right: 250 ms pulse, 200 ms gap, Left: 100 ms pulse); Head Nod: three short vibrotactile pulses at the midline (100 ms pulse, $150 \mathrm{~ms}$ gap, $250 \mathrm{~ms}$ pulse, 200 ms gap, 100 ms pulse); Lean Forward: three vibration motors centered at the midline vibrating simultaneously for $800 \mathrm{~ms}$; Lean Backward: two vibration motors centered at the spine vibrating simultaneously for $800 \mathrm{~ms}$. The experiment involved 11 participants (6 female, 5 male) recruited through the Disability Resource Center at Arizona State University. The age ranges of participants included 22 - 34 (4 participants); 35 - 44 (2); 45 - 54 (2); 55 - 64 (2); and 65 and over (1). Every participant was legally blind: 4 were totally blind, and 7 were visually impaired. 
During the study, each participant engaged in a one-on-one interaction with a trained interviewer. These interactions consisted of thirty minute conversations covering diverse topics (Ex: What is your favorite type of music? Who would you like to see as the next President of the United States?), while an experimenter (wizard) trained in recognizing nonverbal cues, identified these cues from a live video stream, and delivered the cues to the haptic belt. During the interaction, only half of the discussed topics received cues (within-subject design) in an alternating pattern. The sequence of topics was randomized for each participant. Whether the interaction began with the haptic belt turned on or off was randomized. Through a post-experiment questionnaire, participants were asked a series of questions regarding the utility of the belt and the relationship between the nonverbal cues and understanding using a 5point Likert scale from "Low" (1) to "High" (5). Once the study was completed, the Wizard of $\mathrm{Oz}$ experiment was revealed to participants.

Results regarding the utility of the belt were encouraging. Results indicate that the haptic belt was an effective channel of nonverbal communication. The following results are the average responses of all eleven participants as a group: "How easy was it to put on the belt?" $M: 4.0, S D: 1.18$; "How comfortable was the belt?" $M: 4.45, S D$ : 0.69 ; "How easy was it to learn the vibration patterns?" $M: 4.18$, SD: 0.98 ; "How intuitive were the vibration patterns for the nonverbal cues they represented?" $M: 4.0$, $S D$ : 1.00; "When experiencing vibrations with the belt, how easy was it to associate them with the nonverbal cues of the interviewer?" $M: 4.09, S D: 0.94$; and "How easy was it to combine the information received through the vibrations with that of the content of the conversation?" $M: 4.00, S D: 1.0$. These results indicate that the belt was easy to put on and comfortable to wear throughout the duration of the interaction. As well, they indicate that the chosen vibrotactile cues were easy to learn and intuitive for the nonverbal actions they represented.

Participants were also asked if the agreement/disagreement nonverbal cues (as conveyed through the belt) added to their overall conversation (across topics). They used a similar Likert scale as described before to convey how much this addition was: "Do you think that the information presented through the belt added to the conversation?" of which 8 of 11 participants answered yes with $M: 3.38, S D: 1.5$. This data shows that participants felt that agreement/disagreement nonverbal cues added to their interaction. Furthermore, the data is particularly compelling when participants are parsed into groups of totally blind vs. visually impaired. Three out of four totally blind participants responded that 'yes' the belt added to the conversation (M: 4.0, SD: 1.73); while five out of seven participants with some sight responded 'yes', but with M: 3.0, SD: 1.41. Participant understanding per topic was also collected, but more participants are needed to make more conclusive arguments regarding whether or not nonverbal cues of agreement and disagreement, as conveyed though a haptic belt in this study, enhance the perception of an interaction partner's agreement for specific conversation topics by an individual who is blind. 


\section{$5 \quad$ Conclusion and Future Work}

We have explored the usefulness of nonverbal cues for agreement and disagreement during dyadic interactions involving individuals who are blind. A Wizard of Oz experiment was conducted to quickly assess the usefulness of these cues and the utility of the technology. Future work will involve the expansion of this study to include more participants and continued analysis. Using Wizard of $\mathrm{Oz}$ studies, we will explore other nonverbal cues including eye gaze and hand gestures.

Acknowledgements. We would like to thank the National Science Foundation (NSF) and Arizona State University (ASU) for their funding support. This material is partially supported by the NSF under Grant Nos. 1069125 and 1116360 . We would also like to thank Vineeth N. Balasubramanian for his ideas and guidance during the planning of this study.

\section{References}

1. Knapp, M.L.: Nonverbal Communication in Human Interaction. Harcourt College Pub. (1996)

2. Rehman, S.U., Liu, L., Li, H.: Manifold of Facial Expressions for Tactile Perception. In: Proceedings of the IEEE 9th Workshop on Multimedia Signal Processing, pp. 239-242 (2007)

3. Krishna, S., Bala, S., McDaniel, T., McGuire, S., Panchanathan, S.: VibroGlove: An Assistive Technology Aid for Conveying Facial Expressions. In: Proceedings of the CHI 2010 Extended Abstracts on Human Factors in Computing Systems, pp. 3637-3642 (2010)

4. McDaniel, T., Villanueva, D., Krishna, S., Colbry, D., Panchanathan, S.: Heartbeats: A Methodology to Convey Interpersonal Distance through Touch. In: Proceedings of the 28th International Conference Extended Abstracts on Human Factors in Computing Systems, pp. 3985-3990 (2010)

5. McDaniel, T., Krishna, S., Balasubramanian, V., Colbry, D., Panchanathan, S.: Using a Haptic Belt to Convey Non-Verbal Communication Cues during Social Interactions to Individuals who are Blind. In: Proceedings of the IEEE International Workshop on Haptic Audio visual Environments and Games, pp. 13-18 (2008)

6. Martin, B., Hanington, B., Hanington, B.M.: Universal Methods of Design: 100 Ways to Research Complex Problems, Develop Innovative Ideas, and Design Effective Solutions. Rockport Publishers (2012)

7. Bousmalis, K., Mehu, M., Pantic, M.: Spotting Agreement and Disagreement: A Survey of Nonverbal Audiovisual Cues and Tools. In: International Conference on Affective Computing and Intelligent Interaction, vol. 2, pp. 1-9 (2009)

8. Poggi, I.: Mind, Hands, Face and Body: Goal and belief view of multimodal communication. Weidler (2007) 